\title{
A decision tree for the genetic diagnosis of deficiency of adenosine deaminase 2 (DADA2): a French reference centres experience
}

\author{
Mélanie Rama ${ }^{1} \cdot$ Claire Duflos $^{2} \cdot$ Isabelle Melki, ${ }^{3,4}$ Didier Bessis $^{5} \cdot$ Axelle Bonhomme $^{6} \cdot$ Hélène Martin $^{6}$. \\ Diane Doummar $^{7,8} \cdot$ Stéphanie Valence ${ }^{7}$. Diana Rodriguez ${ }^{7,8} \cdot$ Emilie Carme $^{9} \cdot$ David Genevieve $^{10,11}$. \\ Ketil Heimdal ${ }^{12} \cdot$ Antonella Insalaco $^{13} \cdot$ Nathalie Franck $^{14} \cdot$ Viviane Queyrel-Moranne $^{15} \cdot$ Nathalie Tieulie $^{15}$. $^{2}$ \\ Jonathan London ${ }^{16}$. Florence Uettwiller ${ }^{17}$ - Sophie Georgin-Lavialle ${ }^{18}$ - Alexandre Belot ${ }^{19} \cdot$ Isabelle Koné-Paut $^{20}$. \\ Véronique Hentgen $^{21} \cdot$ Guilaine Boursier $^{1} \cdot$ Isabelle Touitou $\mathbb{D}^{1,10}$ - Guillaume Sarrabay ${ }^{1,10}$
}

Received: 27 September 2017 / Revised: 23 February 2018 / Accepted: 27 February 2018 / Published online: 23 April 2018

(c) European Society of Human Genetics 2018

\begin{abstract}
Deficiency of adenosine deaminase 2 (DADA2) is a recently described autoinflammatory disorder. Genetic analysis is required to confirm the diagnosis. We aimed to describe the identifying symptoms and genotypes of patients referred to our reference centres and to improve the indications for genetic testing. DNA from 66 patients with clinically suspected DADA2 were sequenced by Sanger or next-generation sequencing. Detailed epidemiological, clinical and biological features were collected by use of a questionnaire and were compared between patients with and without genetic confirmation of DADA2. We identified 13 patients (19.6\%) carrying recessively inherited mutations in $A D A 2$ that were predicted to be deleterious. Eight patients were compound heterozygous for mutations. Seven mutations were novel (4 missense variants, 2 predicted to affect mRNA splicing and 1 frameshift). The mean age of the 13 patients with genetic confirmation was 12.7 years at disease onset and 20.8 years at diagnosis. Phenotypic manifestations included fever (85\%), vasculitis (85\%) and neurological disorders (54\%). Features best associated with a confirmatory genotype included fever with neurologic or cutaneous attacks (odds ratio [OR] 10.71, $p=0.003$ and $\mathrm{OR} 10.9, p<0.001$ ), fever alone (OR 8.1, $p=0.01$ ), and elevated C-reactive protein (CRP) level with neurologic involvement (OR 6.63, $p=0.017$ ). Our proposed decision tree may help improve obtaining genetic confirmation of DADA2 in the context of autoinflammatory symptoms. Prerequisites for quick and low-cost Sanger analysis include one typical cutaneous or neurological sign, one marker of inflammation (fever or elevated CRP level), and recurrent or chronic attacks in adults.
\end{abstract}

\section{Introduction}

Deficiency of adenosine deaminase type 2 (DADA2) is an autosomal recessive systemic autoinflammatory disorder (SAID) described for the first time in 2014 [1, 2]. Both homozygous or compound heterozygous genotypes have been detected [3]. Although one mutation c.139G>A;p. (Gly47Arg) is frequent, notably in the Georgian population, due to a founder effect, the disease seems to occur

Electronic supplementary material The online version of this article (https://doi.org/10.1038/s41431-018-0130-6) contains supplementary material, which is available to authorized users.

Guillaume Sarrabay

guillaume.sarrabay@inserm.fr

Extended author information available on the last page of the article ubiquitously; indeed, patients with DADA2 have been identified in several countries [4].

The phenotype and outcome observed in DADA2 are quite heterogeneous [5]. Age at disease onset is usually before the second decade of life. The clinical spectrum ranges from single cutaneous lesions to severe systemic inflammatory disease with cerebral complications [6]. Clinical and histopathological features of polyarteritis nodosa (PAN), vasculopathy-related manifestations (myalgia, hypertension and gastrointestinal symptoms), and ischaemic and haemorrhagic strokes are the most frequent DADA2 manifestations [3]. Other clinical presentations include those resulting from immune deficiency and haematological presentations [7-10].

The ADA2 gene, previously named cat eye syndrome chromosome region 1 (CECRI), is located on chromosome $22 \mathrm{q} 11.1$ and has 10 exons. It encodes the adenosine 
deaminase 2 (ADA2) enzyme. ADA2 is $25 \%$ identical to the ADA1 protein according to a BLAST search (https://bla st.ncbi.nlm.nih.gov/). ADA1 is a ubiquitous intracellular enzyme that acts as a monomer [11-13]. It catalyses the irreversible deamination of adenosine and deoxyadenosine in the purine catabolic pathway. ADA1 deficiency is associated with a severe combined immunodeficiency autosomal recessive disease.

ADA2 contains four domains: signal peptide, dimerisation, putative receptor binding, and catalytic domains [3]. The protein is expressed in cells of the myeloid lineage: promonocyte cell lines and monocytes differentiated into macrophages and dendritic cells [11, 12]. ADA2 acts in the extracellular space as an endothelial growth factor [11-13]. ADA2 deficiency may upregulate neutrophil-expressed genes and increase the secretion of pro-inflammatory cytokines [14]. The role of ADA2 in the adaptive immune response remains unclear.

Our autoinflammatory diseases unit in Montpellier University Hospital has been designated as a reference laboratory and is a team of the French reference centre for autoinflammatory diseases. The aim of this study was to describe the clinical characteristics and genotype of a series of patients referred to our laboratory for genetic diagnosis of DADA2 in the context of autoinflammatory symptoms. The objective was to retrospectively identify the symptoms predicting a positive genetic test result, and propose a decision tree to improve DADA2 diagnosis.

\section{Patients and methods}

\section{Study design}

Patients were enrolled when a routine genetic diagnosis was requested. With clinicians of reference centres, we had developed a common clinical form for all SAIDs to be provided with all genetic diagnosis requests. This form collects epidemiological data and includes a list of clinical symptoms and biological markers frequently encountered in SAIDs (see supplementary Figure S1). We retrospectively reviewed these forms for all patients who were screened for $A D A 2$ mutations.

\section{Consent and genetic analysis}

Each participant (or legal guardian if relevant) was fully informed and gave written consent for DNA analysis. Symptomatic cases and, if necessary, some of their relatives were screened for ADA2 mutations (NM_001282225.1). Sanger sequencing $(n=59)$ or next-generation sequencing
(NGS; $n=7$ ) was performed, given the two techniques have proved $100 \%$ concordance when NGS was implemented in the laboratory.

\section{Sanger sequencing}

Two different amplicons were amplified for each exon to circumvent the risk of allele drop-out. Exons 2 to 10 and exon-intron junctions were sequenced in both directions by using ABI PRISM Big Dye Terminator V3.1, the ReadyReaction Cycle-Sequencing kit and ABI 3130 XL (Applied Biosystems).

\section{Next-generation sequencing}

We performed NGS for seven patients (panel of 55 autoinflammatory genes including $A D A 2$; list available upon request, exons 2 to 10 and exon-intron junctions were sequenced). Libraries were prepared by using SureSelect Target Enrichment Capture custom kits (Agilent). Sequencing reactions involved MiSeq or NextSeq500 equipment (Illumina).

\section{Quantitative PCR (qPCR)}

When relevant (e.g., when a single clearly pathogenic variant was detected by sequencing or with apparent homozygosity), real-time quantitative PCR (qPCR) was performed for each coding exon (2 to 10) by using a LightCycler 480 thermocycler (Roche) to search for a possible small rearrangement of the second allele.

\section{Interpretation}

Variants were analysed by using Seqscape (Applied Biosystem), Seqnext (JSI) and standard in silico tools. These latter tools included the Alamut pipeline (Interactive Biosoftware) for missense mutations (GVGD, SIFT, Polyphen2, MutationTaster) and MaxEntScan (MES), Human splice finder (HSF), and neural network splice (NNSplice) for splice mutations.

\section{Statistical analysis}

Data on clinical symptoms and biological markers were extracted from our standard clinical form for genetic requests related to autoinflammatory diseases (supplementary Figure S1). The association between these potential diagnostic items and genetic confirmation of the disease was estimated by odds ratios (ORs), 95\% confidence intervals (CIs) and Fisher exact test. 


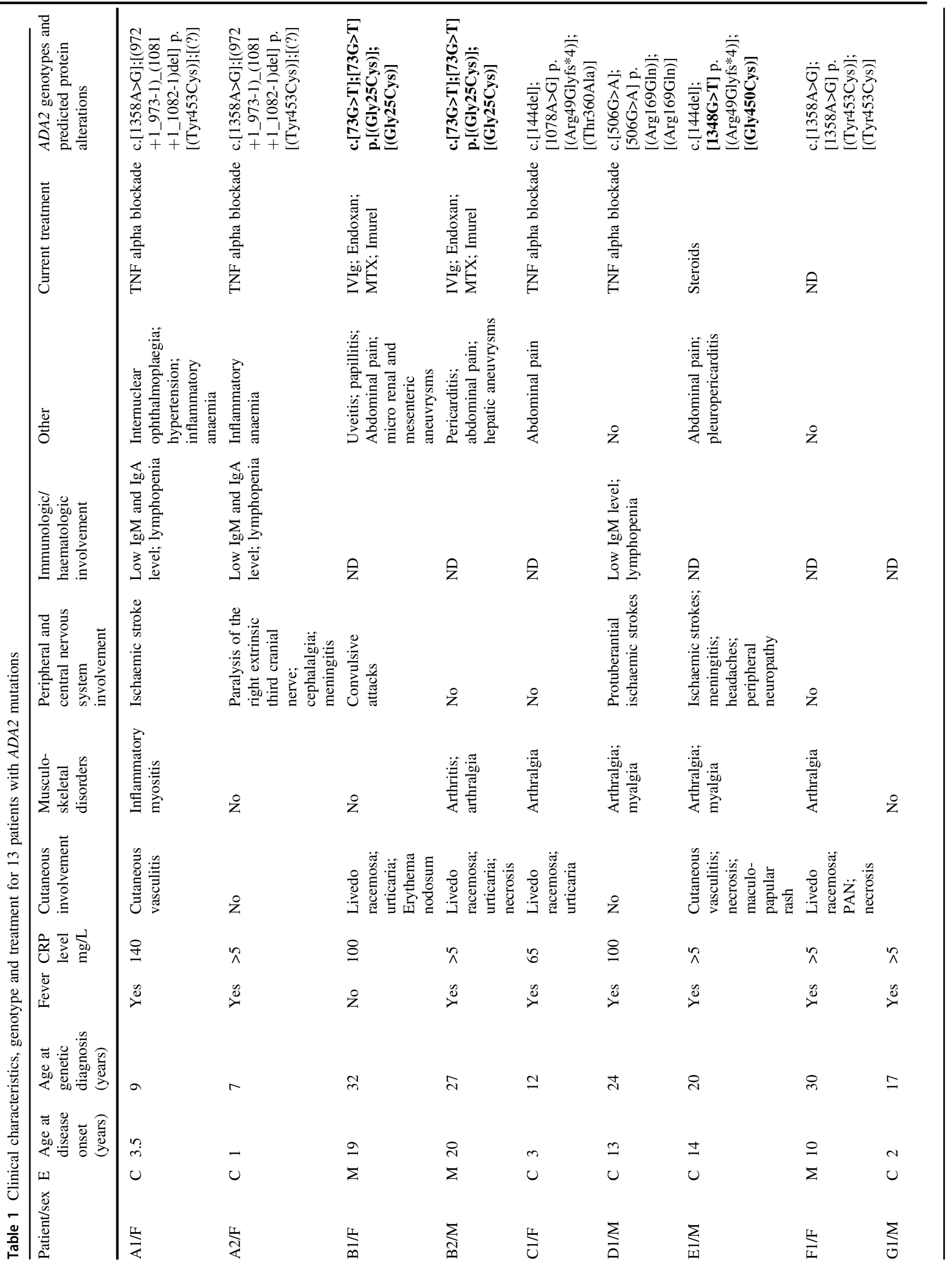




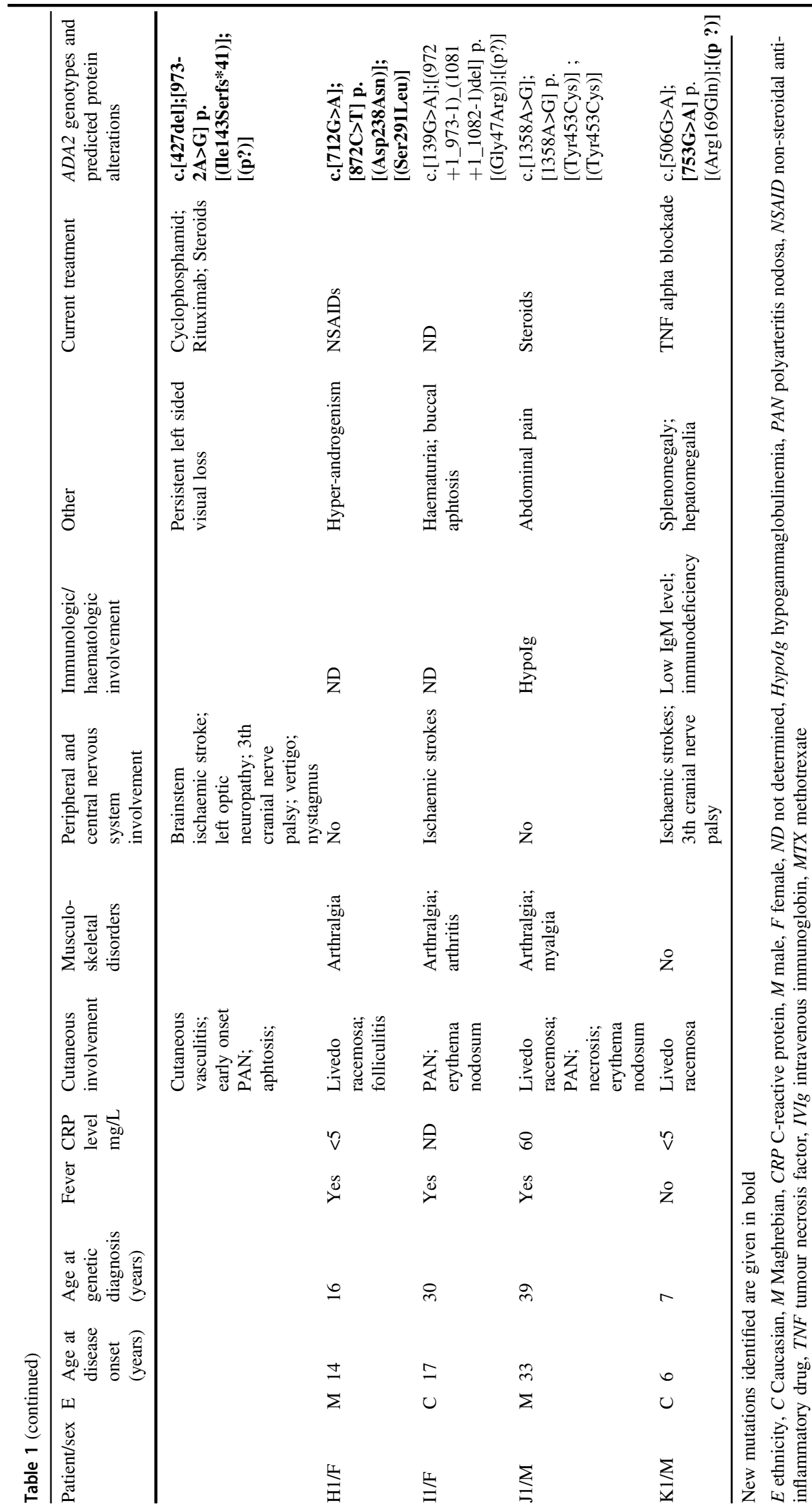


a) In Silico tools predictions for the newly identified variants

\begin{tabular}{|c|c|c|c|c|c|c|c|c|c|c|c|c|c|c|c|c|c|}
\hline $\begin{array}{l}\text { gDNA } \\
\text { position }\end{array}$ & Ref & Alt & $\begin{array}{l}\text { CDNA } \\
\text { position }\end{array}$ & Ref & Alt & $\begin{array}{l}\text { Protein } \\
\text { position }\end{array}$ & Ref & Alt & ExAC & PolyPhen & $\begin{array}{l}\text { PolyPhen } \\
\text { Value }\end{array}$ & SIFT & $\begin{array}{l}\text { SIFT } \\
\text { Value }\end{array}$ & $\begin{array}{l}\text { CADD } \\
\text { (PHRED) }\end{array}$ & MaxEnt & HSF & NNSplice \\
\hline 17662804 & C & A & 1348 & G & T & 450 & Gly & Cys & $0.0012 \%$ & $\begin{array}{l}\text { probably } \\
\text { damaging }\end{array}$ & 1 & deleterious & 0 & 27.8 & NA & NA & NA \\
\hline 17669339 & $\mathrm{~T}$ & C & $973-2$ & $A$ & G & NA & NA & NA & $0.012 \%$ & NA & NA & NA & NA & 24.3 & $-100 \%$ & $-100 \%$ & $-100 \%$ \\
\hline 17672582 & G & A & 872 & C & T & 291 & Ser & Leu & $0.00041 \%$ & $\begin{array}{l}\text { probably } \\
\text { damaging }\end{array}$ & 0.974 & deleterious & 0 & 25.8 & NA & NA & NA \\
\hline 17684453 & C & $\mathrm{T}$ & 753 & G & A & 251 & Pro & Pro & $0.0024 \%$ & NA & NA & NA & NA & 9.957 & $-48.9 \%$ & $-11.5 \%$ & $-60 \%$ \\
\hline 17684494 & c & T & 712 & G & A & 238 & Asp & Asn & NA & $\begin{array}{l}\text { probably } \\
\text { damaging }\end{array}$ & 1 & deleterious & 0 & 27.5 & NA & NA & NA \\
\hline 17690495 & C & A & 73 & G & T & 25 & Gly & Cys & $0.0016 \%$ & benign & 0.003 & tolerated & 0.13 & 8.138 & NA & NA & NA \\
\hline 17688076 & 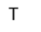 & - & 427 & A & - & 143 & Ile & Serfs $* 41$ & NA & NA & NA & NA & NA & 17.36 & NA & NA & NA \\
\hline 17687992 & G & A & 511 & c & T & 171 & Arg & Trp & $0.12 \%$ & benign & 0.067 & deleterious & 0 & 19.56 & NA & NA & NA \\
\hline
\end{tabular}

b) Adenosine deaminase type 2 gene (ADA2, NM_001282225.1) with already published and newly identified variants

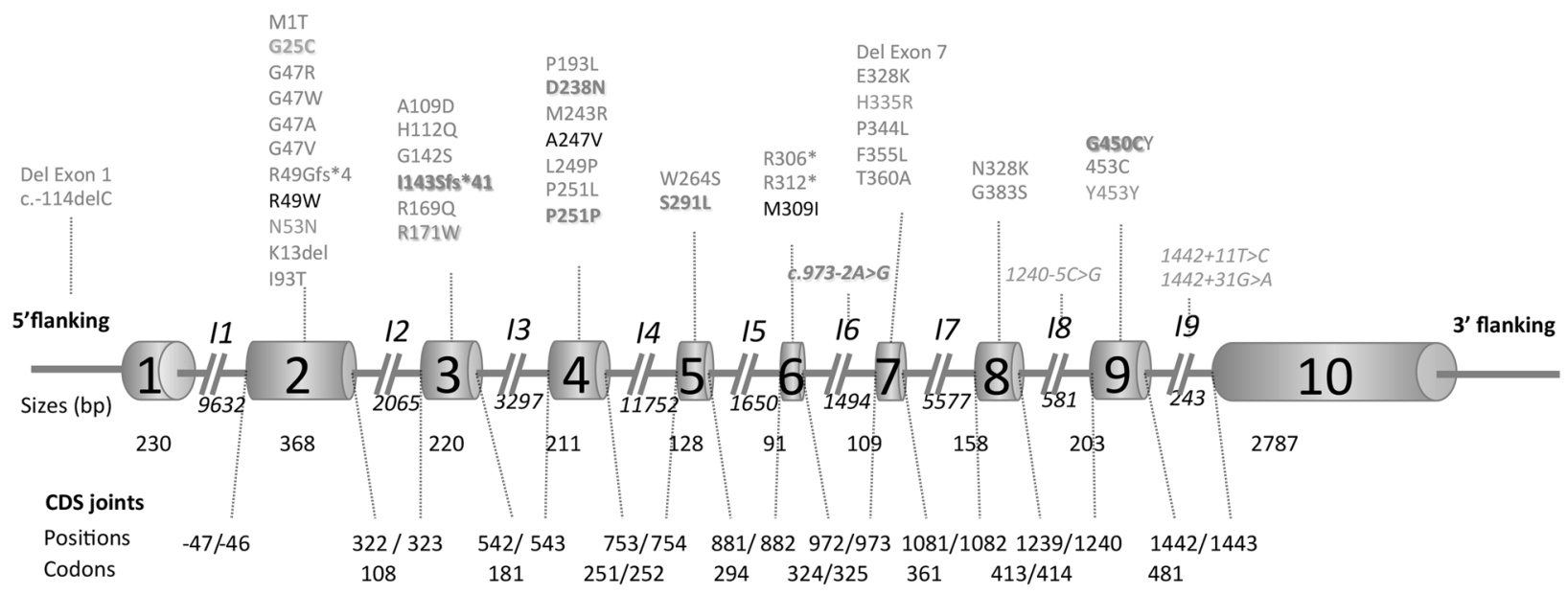

Fig. 1 The adenosine deaminase type 2 (ADA2) genes and novel sequence variants. a In silico tool predictions for the newly identified variants. gDNA genomic position on human genome $\mathrm{HG}$ 37, Ref reference allele, Alt new allele, cDNA coding sequence position, prediction using the combined annotation dependent depletion (CADD) in silico tool cadd.gs.washington.edu, HSF Human Splicing Finder, MES MaxEntScan, NNS Neuronal Network Slipce, NA not

Then, we estimated the percentage of correctly classified cases (a classical measure of accuracy) and the sensitivity and specificity of each of the diagnostic elements. This methodology was repeated to examine the association and diagnostic values of various combinations of these items. Highly associated combinations of DADA2 clinical symptoms and biological markers were chosen as potential prerequisites for the decision tree. As a preliminary validation procedure, we applied these prerequisite items to an external sample of all published cases of genetically confirmed DADA2 whenever our items were available in reports and to an internal series of all patients with a genetically confirmed AID other than DADA2, referred since the implementation of the present version of our clinical form (supplementary Figure S1). applicable. b Schematic representation of the adenosine deaminase type 2 ( $A D A 2)$ gene (adapted from Infevers). In bold: newly identified DADA2 variants, in red: pathogenic DADA2 variants, in orange: variant of unknown clinical significance (VUS), in green: common polymorphism, in black: Behçet susceptibility factors. This figure shows the variant usual name (i.e., as first published). Bp Base pair, CDS CoDing Sequence

\section{Results}

\section{Demographic data}

Requests for genetic diagnosis of DADA2 have greatly increased since 2014. Our series includes all patients $(n=$ 66) who were referred to our laboratory for clinical suspicion of DADA2. The referring clinicians were from various medical specialties: 33 paediatricians [paediatric rheumatology $(n=13)$, generalist paediatrics $(n=11)$, paediatric neurology $(n=6)$ and paediatric haematology $(n=3)]$ and 33 clinicians for adults [internal medicine $(n=20)$, dermatology $(n=9)$ genetics $(n=3)$ and nephrology $(n=1)]$. Patients were of European Caucasian $(n=35)$, Maghrebian $(n=19)$, Middle East $(n=5)$, African $(n=3)$, Jewish $(n=$ $3)$ or Asian ancestries $(n=1)$. Only two families had more 
than one symptomatic member (Families A and B, Figure $\mathrm{S} 2$ in supplementary file). Consanguinity was reported in two families (B and F). The male to female ratio was 0.91 . The mean age at disease onset was 14.0 years (median 10 years, min-max: 4 months-69 years, standard deviation (SD): 14.4 years).

\section{ADA2 mutations}

DADA2 was confirmed in $13(19.6 \%)$ of the 66 patients from 11 unrelated families (Table 1). We found 8 missense and 5 non-sense different mutations. In all families but family J, DNA from relatives was available and the variants could be confirmed to be located in trans. Eight patients were compound heterozygous and five were homozygous for mutations c.73G $>$ T;p.(Gly25Cys), c.506G $>\mathrm{A}$;p. (Arg169Gln) or c.1358A $>$ G;p.(Tyr453Cys). Six variants had previously been associated with DADA2: c.144del;p. (Arg49Glyfs*4), c.139G>A;p.(Gly47Arg), c.506G>A;p. (Arg169Gln), c.1358A>G;p.(Tyr453Cys), c.1078A $>$ G;p. (Thr360Ala) and deletion of exon 7 [7, 15-18]. Seven novel mutations were found in families B, E, G, H and K (Fig. 1). In silico tools predicted that two novel variants, c.973$2 \mathrm{~A}>\mathrm{G}$ and c.753G $>\mathrm{A}$, may affect mRNA splicing (Fig. 1a). Mutation c.973-2A $>\mathrm{G}$ is a rare canonical splicing variant absent in the ExAC (http://exac.broadinstitute.org) and dbSNP databases (https://www.ncbi.nlm.nih.gov/projects/ $\mathrm{SNP} /$ ). It is predicted to alter the wild-type acceptor site (>30\% impact according to $\mathrm{HSF}$ and $58 \%$ according to MES). The second variant, c. $753 \mathrm{G}>\mathrm{A}$, is a substitution, which apparently does not change codon 251 . However, this guanine is the last nucleotide of exon 4 and is located within a donor splicing consensus site. Hence, this mutation is predicted to result in a truncated protein.

We identified one new frameshift mutation, c.427delA;p. (Ile143Serfs*41), and 4 novel missense variants: c.73G $>\mathrm{T}$; p.(Gly25Cys), c.1348G>T;p.(Gly450Cys), c.712G>A;p. (Asp238Asn) and c.872C $>$ T;p.(Ser291Leu). Two consanguineous siblings, B1 and B2, were homozygous for $\mathrm{p}$. (Gly25Cys) and presented the same phenotype. They had severe pleomorphic vasculitis features (PAN, livedo, renal microaneurysms) with inflammation. Their parents were asymptomatic and heterozygous for this variant, which moreover was absent from general databases. For this reason and although the pathogenicity score obtained for this variant was conflicting, we considered it a disease-causing mutation. In family E, p.(Gly450Cys) was predicted to have deleterious effects on the protein function. The glycine located at position 450 in the catalytic domain of the $A D A 2$ protein is well conserved across species, and this variant is absent from ExAC or 1000Genome databases. A young female of Maghrebian origin (family H1) had a history of
Table 2 Clinical characteristics of the patients with and without genetically confirmed DADA2

\begin{tabular}{|c|c|c|c|c|}
\hline & \multicolumn{2}{|c|}{$\begin{array}{l}\text { Unconfirmed } \\
\text { DADA2 }\end{array}$} & \multicolumn{2}{|l|}{$\begin{array}{l}\text { Confirmed } \\
\text { DADA2 }\end{array}$} \\
\hline Disease course & \multicolumn{2}{|c|}{$\begin{array}{l}\text { Age, years } \\
\text { (mean/median) }\end{array}$} & \multicolumn{2}{|c|}{$\begin{array}{l}\text { Age, years } \\
\text { (mean/median } \\
\text { age) }\end{array}$} \\
\hline Onset & $14.0(9)$ & - & $12.0(13)$ & - \\
\hline Diagnosis & - & - & $20.8(20)$ & - \\
\hline Clinical signs & $\mathrm{n}(\mathrm{N})$ & $\%$ & $\mathrm{n}(\mathrm{N})$ & $\%$ \\
\hline CRP level $\geq 5 \mathrm{mg} / \mathrm{dL}$ & $25(44)$ & 56.8 & $10(12)$ & 83.4 \\
\hline Fever $\geq 38^{\circ} \mathrm{C}$ & $16(45)$ & 35.5 & $11(13)$ & 84.6 \\
\hline Neurologic involvement & $17(50)$ & 34 & $7(13)$ & 53.8 \\
\hline Stroke & 15 & - & 6 & - \\
\hline Intracranial haemorrhagia & 1 & - & 0 & - \\
\hline PNS involvement & 3 & - & 4 & - \\
\hline Other & 6 & - & 2 & - \\
\hline Ophthalmologic involvement & $2(50)$ & 4 & $3(13)$ & 23.1 \\
\hline Hepato-splenomegaly & $15(50)$ & 30 & $1(13)$ & 7.7 \\
\hline Musculoskeletal disorder & $24(50)$ & 48 & $9(13)$ & 69.2 \\
\hline Cutaneous involvement & $37(50)$ & 74 & $11(13)$ & 84.6 \\
\hline Livedo racemosa & 9 & - & 7 & - \\
\hline Ulcerations of extremities & 3 & - & 4 & - \\
\hline Vasculitis & 28 & - & 2 & - \\
\hline Maculopapular rash & 15 & - & 1 & - \\
\hline Immunodeficiency & $7(50)$ & 14 & $5(13)$ & 38 \\
\hline Low immunoglobulin level & 5 & - & 5 & - \\
\hline Lymphopenia & 3 & - & 3 & - \\
\hline Recurrent infections & $2(50)$ & 4 & 0 & 0 \\
\hline
\end{tabular}

CRP C-reactive protein, PNS peripheral neurological system, Other meningoencephalitis or meningitis or epilepsy. $n$ number of affected patients, $N$ number of patients with complete clinical form

recurrent livedo racemosa and folliculitic rashes accompanied by fever since age 14 . At age 16 , she had musculoskeletal involvement with inflammatory arthralgia and biological hyperandrogenism. DADA2 was suspected despite the absence of neurological attacks or increased Creactive protein (CRP) level. ADA2 sequencing revealed two novel variants: p.(Asp238Asn) and p.(Ser291Leu). Both are located in the catalytic domain of the protein in exons 4 and 5, respectively, and are highly conserved across species. They were both predicted to be probably damaging to the protein function.

\section{Identifying symptoms in genetically confirmed patients}

Demographic features and clinical presentations of the 13 patients with genetically confirmed DADA2 are in Table 1. The mean age at disease onset was 12.0 years (min-max: 1-33, SD: 9.1) and mean age at diagnosis 20.8 years 
Table 3 Sensitivity and specificity of biological and clinical characteristics in 13 patients with and without genetically confirmed DADA2

\begin{tabular}{llll}
\hline Clinical disease & Se $[95 \% \mathrm{CI}]$ & Sp $[95 \% \mathrm{CI}]$ & OR $[95 \% \mathrm{CI}]$ \\
\hline Fever + CRP + NI & $0.42[0.14 ; 0.70]$ & $0.95[0.89 ; 1.01]$ & $13.9[1.86 ; 172.87]$ \\
Fever + CRP + CI & $0.58[0.30 ; 0.86]$ & $0.82[0.71 ; 0.93]$ & $6.04[1.28 ; 31.44]$ \\
Fever + NI + CI & $0.31[0.05 ; 0.57]$ & $0.98[0.94 ; 1.02]$ & $17.72[1.53 ; 955.70]$ \\
Fever + NI & $0.46[0.18 ; 0.74]$ & $0.93[0.86 ; 1.00]$ & $10.71[1.81 ; 82.45]$ \\
Fever + CI & $0.69[0.43 ; 0.95]$ & $0.84[0.73 ; 0.95]$ & $10.9[2.37 ; 61.47]$ \\
CRP + NI + CI & $0.25[0.01 ; 0.50]$ & $0.93[0.86 ; 1.00]$ & $4.29[0.49 ; 37.64]$ \\
CRP + NI & $0.42[0.14 ; 0.70]$ & $0.91[0.83 ; 0.99]$ & $6.63[1.13 ; 43.07]$ \\
CRP + CI & $0.67[0.40 ; 0.94]$ & $0.63[0.49 ; 0.77]$ & $3.26[0.75 ; 17.02]$ \\
CI + NI & $0.31[0.05 ; 0.57]$ & $0.77[0.65 ; 0.90]$ & $1.45[0.27 ; 6.57]$ \\
Fever & $0.85[0.65 ; 1.05]$ & $0.6[0.46 ; 0.74]$ & $8.1[1.50 ; 84.40]$ \\
CRP level $\geq 5$ mg/dL & $0.83[0.62 ; 1.04]$ & $0.46[0.31 ; 0.61]$ & $4.21[0.76 ; 44.27]$ \\
NI & $0.54[0.26 ; 0.82]$ & $0.33[0.19 ; 0.47]$ & $0.59[0.14 ; 2.53]$ \\
CI & $0.69[0.43 ; 0.95]$ & $0.44[0.29 ; 0.59]$ & $1.78[0.42 ; 9.12]$ \\
Musculoskeletal disorders & $0.69[0.43 ; 0.95]$ & $0.61[0.47 ; 0.75]$ & $3.49[0.82 ; 18.05]$ \\
\hline
\end{tabular}

Se Sensitivity, $S p$ Specificity, OR odds ratio, 95\% CI confidence interval, $C R P$ C-reactive protein, $N I$ neurologic involvement such as ischaemic or haemorragic stroke or peripheral palsy, $C I$ cutaneous involvement such as livedo racemosa, nodular rash, erythema nodosum, vasculitis and necrosis

$* p=0.05-0.005, * * p=0.005-0.001, * * * p<0.001$

(min-max: 7-39, SD: 10.4) (Table 2). A few patients had a particular disease course. One patient (J1) had late clinical manifestations at age 33, with a cutaneous phenotype and an immunological disorder. Patient D1 was first diagnosed with juvenile idiopathic arthritis; typical DADA2 manifestations, such as ischaemic stroke, occurred secondarily.

Among the 13 patients with confirmed DADA2, fever was present in $11(85 \%)$ and elevated CRP level in 10 (Table 2). Eleven patients showed cutaneous involvement, including livedo racemosa, nodular rash, vasculitis (PAN), erythema nodosum or peripheral necrosis. Musculoskeletal manifestations concerned 9 patients (69\%). Seven patients (54\%) presented peripheral and/or central nervous system involvement such as ischaemic and/or haemorrhagic stroke or peripheral nerve palsy. Five patients $(38 \%)$ had a history of recurrent infection, immunodeficiency and/or hypogammaglobulinemia. Immunologic deficiency was always associated with other symptoms in families $\mathrm{A}, \mathrm{D}, \mathrm{J}$ and $\mathrm{K}$.

\section{Clinical characteristics of patients with no confirmatory genotype}

Three patients without a family history of DADA2 were heterozygous for an $A D A 2$ variant (supplementary Table S3). One presented a variant of uncertain significance (VUS) with discordant in silico predictions, and one presented a benign missense variation. Both presented few DADA2 clinical features. Patient M1 carried the known p. (Ala247Val) variant;[19] symptoms occurred at age 1 year. Raynaud's syndrome was the only clinical sign indicated by the clinician requesting $A D A 2$ sequencing. There were no other DADA2 characteristics such as immunologic deficiency or cutaneous involvement or clinical inflammation during episodes or increased CRP level. Patient N1, from Algeria, had a missense variant, c.511C >T;p.(Arg171Trp), that we considered a polymorphism because of high minor allele frequency of $1.5 \%$ in individuals of African origin according to ExAC (Fig. 1a). The symptoms had begun at age 5 years and included oral aphtosis, myalgia and increased CRP level during flares. No neurological episode was reported. The third patient (L1) had symptoms more consistent with DADA2. Disease began at age 5 with a discrete inflammatory syndrome including fever and CRP level increased to $27 \mathrm{mg} / \mathrm{dL}$. The accompanying signs were cephalalgia, arthralgia and myalgia, papular rash with pruritis and erratic gastrointestinal manifestations (especially diarrhoea). Only one variant, p.(Gly47Arg), was found on conventional sequencing analysis. This variant was known to be clearly pathogenic $[3,16]$. Although the hypothesis of a copy-number variation was ruled out on qPCR, a second disease-causing variant affecting the gene's promoter or non-coding regulatory sequences may exist. However, ADA2 activity measurement (not shown) revealed an intermediate profile, consistent with the phenotype.

We detected no disease-causing mutation in $A D A 2$ in the remaining 50 patients Table 2). The mean age at disease onset was 14.0 years (min-max: 4 months-69 years, SD: 15.3). Fever and elevated CRP level were observed in $35.5 \%$ and $56.8 \%$ of the patients, respectively. Cutaneous 
involvement was also a predominant clinical feature, but neurologic symptoms were less frequent. Fifteen patients presented stroke, one patient intracranial haemorrhagia and three patients peripheral neuropathy.

\section{Comparison of patients with and without genetically confirmed DADA2}

Phenotypes of patients with and without genetically confirmed DADA2 were compared (Table 3). Fever was more frequent in patients with than without genetic confirmation $(\mathrm{OR}=8.1, p=0.01)$. As well, cutaneous and neurological signs were significantly more frequent when associated to fever. Elevated CRP level was the biological sign with the best sensitivity (83\%) and specificity (46\%). The other characteristics taken alone were not contributive. We then evaluated the performance of combined symptoms. The association of a marker of inflammation (fever or CRP level) with skin or neurological manifestations enhanced the odds of a confirmatory genotype, for example, elevated CRP level combined with central ischaemic and haemorrhagic involvement, or peripheral neuropathy (OR 6.63, $p$ $=0.017)$. The association of three clinical characteristics further increased this performance, which was the best for fever and neurological and cutaneous disorders (OR 17.72, $p=0.008$ ), and for inflammation markers (fever and CRP) and either of the DADA2 typical features such as ischaemic stroke or livedo racemosa (OR $>6, p \leq 0.01)$. Fig. 2 highlights that more than $65 \%$ of the patients were misclassified when considering CRP, musculoskeletal, neurological, cutaneous signs or fever individually. Altogether, the association of two or three clinical signs improved the proportion of cases correctly classified $(>80 \%)$.

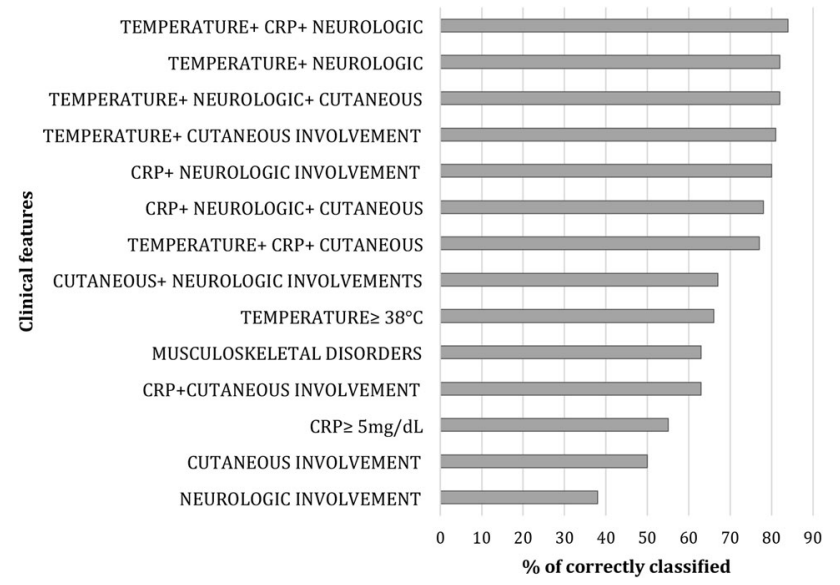

Fig. 2 Percentage of cases correctly classified (CCR). The combined or isolated items are classified from highest to lowest according to their likelihood of being associated with a confirmatory genetic diagnosis of DADA2. CRP C-reactive protein level increased up to $5 \mathrm{mg} / \mathrm{dL}$ during an episode

\section{A proposed decision tree for genetic diagnosis of DADA2}

As shown previously, a number of cutaneous or neurological signs and inflammation (fever or elevated CRP level) were the identifying symptoms that when combined were best associated with genetic confirmation of the DADA2 diagnosis. All of our 13 patients with genetic confirmation had more than three episodes of systemic inflammation. To better rule out a non-hereditary origin of the phenotype, we suggest observing at least one recurrence or chronic evolution in adults before requesting molecular investigation. In children, the evolution may be dramatic, and a relevant diagnosis may be an emergency.

To validate the items described as possible prerequisites for gene-targeted (Sanger) genetic diagnosis, we tested them in all published cases of genetically confirmed DADA2 with enough data $(n=52)[3,16,20]$. Two paediatric cases did not fulfil the prerequisites. One boy presented at age 5 with recurrent fever, splenomegaly, generalised lymphadenopathy, increasing levels of acute-phase reactants, anaemia, thrombocytosis and polyclonal hyperimmunoglobulinemia [21]. The other boy was diagnosed at age 6 with fever, hypogammaglobulinemia, arthralgia and hepatosplenomegaly [20]. However, our NGS panel would have identified both patients.

We also tested these prerequisites in a series of 53 patients with other SAIDs that we genetically confirmed in our lab, notably, familial mediterranean fever (FMF) $(n=$ 32), mevalonate kinase deficiency $(n=5)$, A20 haploinsuffisancy $(n=3)$, tumour necrosis factor receptorassociated periodic syndrome $(n=3)$, and cryopyrinassociated periodic syndrome $(n=1)$. Only one patient met the prerequisites and would have been eligible for ADA2 testing. He was homozygous for c.2080A>G;p. (Met694Val) and had severe FMF and PAN, a well-known complication of this disease.

These studies led to the identification of a minimal common clinical set of symptoms in positive patients. We propose a provisional decision tree (Fig. 3) that should help define optimised conditions predicting a positive genetic analysis.

\section{Discussion}

We report a large series of patients referred to us for genetic diagnosis of DADA2. We used information provided by the ordering clinicians to (1) describe the population with suspected DADA2, (2) compare our patients to those previously reported and (3) try to delineate prerequisites for a positive genetic diagnosis. We identified 13 patients carrying recessively inherited mutations in $A D A 2$ that were predicted to be deleterious. Eight patients were compound 
Fig. 3 Decision tree for genetic diagnosis of DADA2. At the top of the figure are the selected prerequisites for a genetic diagnosis. At least one item of each of inflammation, vasculitis and clinical course must be present for Sanger sequencing. Bold lines depict advised steps. Dotted lines show optional decisions. CRP C-reactive protein, $A D A 2$ adenosine deaminase type 2, SAID systemic autoinflammatory disorder, NGS next-generation sequencing. *Livedoid skin rash, vasculitis, periarteritis nodosa, erythema nodosum, necrosis of extremity. $* *$ central or peripheric neurologic involvement, ischaemic, haemorrhagic or palsy

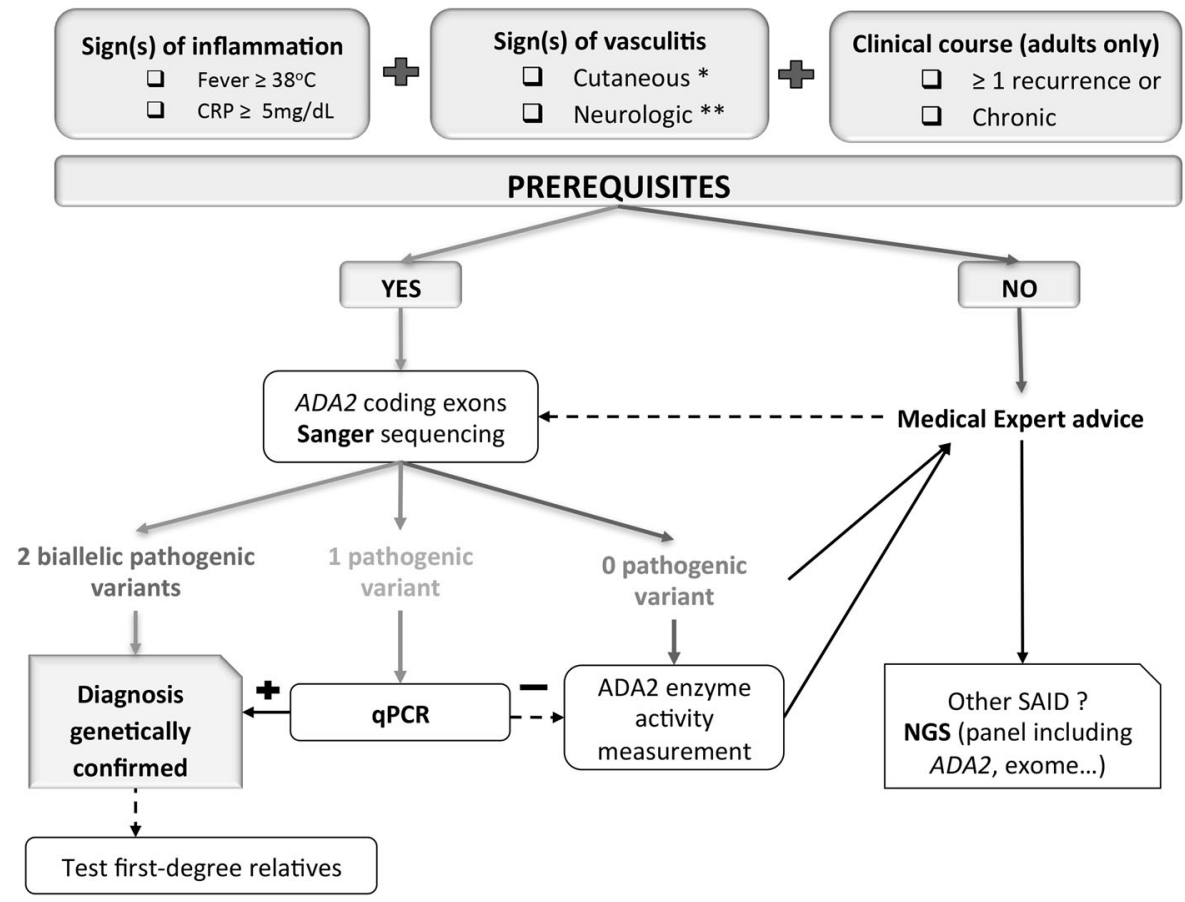

heterozygous for mutations. Seven mutations were novel (4 missense variants, 2 predicted to affect mRNA splicing and 1 frameshift). Phenotypic manifestations included fever, vasculitis and neurological disorders. Prerequisites for quick and low-cost Sanger analysis included one typical cutaneous or neurological sign, one marker of inflammation (fever or elevated CRP level), and recurrent or chronic attacks in adults.

We describe a large spectrum of disease expression and severity, ranging from limited cutaneous vasculitis to severe cerebral vasculitis, in agreement with previous reports. Our SAID clinical form revealed novel symptoms at DADA2 presentation. For example, patient D1 had neither vasculitis nor neurologic involvement at first. His initial symptoms were arthritis symptoms. Musculoskeletal disorders (arthritis, arthralgia or myalgia) accompanied more specific symptoms in nine patients $(69 \%$ of patients with genetic confirmation of DADA2) and were not necessarily associated with vasculitis. The importance of rheumatologic involvement was not highlighted in previous series and suggests that patients with undiagnosed DADA2 may consult in rheumatologic departments. Caorsi et al. also hypothesised that DADA2 might represent an unrecognised condition in adult patients consulting rheumatologists [20]. The age at disease onset in our study group was twice later than in published paediatric series, (mean 12.7 vs 5.3 years; median 13.5 vs 3 years). Seven of our patients were indeed recruited in adult departments. One patient (J1) had a very late and severe dermatological disease, with inaugural necrosis at age 33 . These symptoms could account for the apparent later disease onset of our patients.
Our study expands the spectrum of known DADA2associated mutations recorded in the Infevers registry of hereditary autoinflammatory-disorder mutations [19]. Indeed, we identified 7 novel mutations: 4 missense, 1 frameshift and 2 splicing variants associated with typical DADA2 symptoms. Figure $1 \mathrm{~b}$ highlights that DADA2 mutations are distributed all along the gene, with two mutational hot spots at codon 47 (four different mutations) and codon 251 ( 2 mutations). It also shows that exonic deletions may occur, thereby justifying the use of qPCR when only one pathogenic mutation is identified in a patient with a clear DADA2 phenotype [18, 22].

We found two allelic pathogenic ADA2 mutations in one-fifth of our patients, thus confirming the DADA2 diagnosis. Three patients had heterozygous mutations (Table 1S). Two presented one VUS: c.740C $>$ T; p. (Ala247Val) and c.511C $>\mathrm{T}$; p.(Arg171Trp), respectively. Because the clinical features of these patients included none of the DADA2 features of vasculitis, systemic inflammation, immunodeficiency or neurological manifestations, the suspicion of this diagnosis was considered too weak to extend the ADA2 analysis, and the physicians considered that their patients had another, still undefined, SAID. The genotype p.(Gly47Arg);(Gly47=) found in the third heterozygous patient was probably responsible for the mild phenotype, as supported by the reduced but not null enzyme activity. A recent study showed that ADA2 heterozygote patients exhibit mild symptoms such as livedo, arthromyalgia, and recurrent infections [20]. This work and ours support the hypothesis of a gene dosage effect accounting for the variable clinical expression observed in patients with 
DADA2-like disease, as previously demonstrated in other autoinflammatory diseases [23].

Phenotypic variability is common in DADA2 $[3,9]$. Our series is too small to detect a definitive or novel genotype-phenotype correlation. However, we could confirm some trends. Our two patients who were homozygous for the pathogenic p.(Tyr453Cys) variant (patients F1 and J1) had a cutaneous presentation and were referred in the third decade of life by a dermatologist. Two other patients who were heterozygous for this variant (patients $\mathrm{A} 1$ and A2) had also cutaneous signs. All reported patients carrying this mutation had livedoid skin rash [3]. Two patients (D1 and K1) presenting the p.(Arg169Gln) variant, one homozygous and one heterozygous, had hypogammaglobulinemia, a defect frequently associated with this variant $(62 \%)$ [3]. While preparing this manuscript, Schepp et al. published data for a cohort of 181 adult patients with immunodeficiency or hypogammaglobulinemia as a common failure. The authors' NGS analysis (large panel or exome) highlighted $2 A D A 2$ pathogenic variants in 11 patients [8]. Vascular manifestations and non-infectious fever were present in $64 \%$ of his patients, demonstrating two clinical presentations, which might overlap in some patients. It also confirms that immunodeficiency seems a more common trait of the disease in adults than previously anticipated. Of note, 5 of 11 patients carried the p.(Arg169Gln) variant.

At least six other large DADA2 series have been reported $[1-3,9,16,24]$. The inclusion criteria and study design were variable, according to the goal of the study. The two initial papers described the identification of the gene in patients with recurrent stroke [1] or PAN [2]. Two studies preferred clinical criteria and analysed the prevalence of ADA2 mutations in patients with a typical DADA2 phenotype $[3,24]$. Two opted for a genetic criterion and discussed presymptomatic diagnosis and variable expression, respectively $[9,16]$. We took a different strategy. The aim of our study was to compare patients with a clearly pathogenic genotype to those with no genetic confirmation in order to identify the clinical constellation most likely to lead to genetic confirmation. We imposed no clinical selection or criteria before testing but collected uniform clinical data for each patient. Therefore, this series exactly reflects the context of requests for sequencing the ADA2 gene we receive in our laboratory.

In our series, the best performance resulted from the combination of biological and clinical signs (Table 3). We propose the decision tree illustrated in Fig. 3. The first mandatory prerequisite we suggest is fever (or at least elevated CRP level) because this clinical sign, alone or in combination with other symptoms, was a significant marker of genetic confirmation. We also advise associating any one of the following signs of vasculitis: PAN, livedoid skin rash or systemic vasculitis such as that involving the cerebral or peripheral neurologic system because the clinical symptoms may differ among patients. In addition, we estimate that a chronic or recurrent clinical course is an important criterion to decrease the risk of sporadic causes of inflammation in adults. All patients with genetically confirmed DADA2 had at least three flares; therefore, we consider it reasonable to require at least one recurrence as a condition for genetic analysis.

We include two additional items in this decision tree that we did not evaluate formally. We do not require reduced enzymatic activity as a condition for genetic analysis. However, measurement of ADA2 activity likely represents an added value to the diagnosis, because serum ADA2 enzyme activity was significantly lower in all confirmed DADA2 cases than in healthy controls, even in the absence of ADA2 mutation [3]. Nanthapisal et al. strongly recommended screening first-degree relatives because presymptomatic molecular diagnosis of DADA2 may allow for early treatment in the event of an acute presentation, so we retain this suggestion. We do test symptomatic relatives and plan to test asymptomatic relatives on request also. Finally, we could not evaluate cytopenia and hypogammaglobulinemia as possible prerequisites, because these items are not present in our clinical form. However, these data could be extracted in 5 of $13(38 \%)$ of our confirmed patients for whom the space "other symptom" was used. This finding is consistent with previous data (33-55\%) [20]. Moreover, Caorsi et al. observed no difference in incidence of hypogammaglobulinaemia by mutation status of patients [3]. Therefore, this item is probably optional in our proposed decision tree.

Our model performed well retrospectively. Two paediatric patients would have been missed by using only the proposed prerequisites for Sanger sequencing [20, 21]. Their outcome is unknown. They could show a complete phenotype in later ages. Diagnosis in childhood may be an urgent matter, and delaying molecular investigation in children not fulfilling our prerequisites seems not advisable. On the other hand, our decision tree encompasses this risk by clearly suggesting medical expert advice, with possible NGS including this gene. However, our decision tree would not have resulted in too much testing either. Indeed, a simulation showed that unnecessary genetic analysis of ADA2 would have been performed for only $1.9 \%$ of SAID patients without DADA2.

In summary, this work demonstrated that our patients with DADA2 in France present clinical features similar to those of patients from different countries. We also contribute to expanding the mutational spectrum associated with the DADA2 phenotype. Finally, we used systematic and homogeneous clinical forms along with genetic testing orders to highlight the items most frequently encountered in patients with a confirmed genotype. We propose a preliminary decision tree for genetic diagnosis of DADA2 in 
the context of SAIDs and plan to confirm this algorithm in prospective cohorts. We believe that it may already help physicians prioritise molecular screening among SAID patients with possible DADA2 disease.

Acknowledgements We thank Mrs Cécile Rittore and M. Bruno Dumont for excellent technical contribution. We thank Laura Smales (http://www.biomedediting.com), for editing the manuscript for English language. The French Ministry of Health and the University Hospital of Montpellier supported this work.

Author contributions MR, IT and GS conceived and designed the study. CD provided help for statistical analysis and design of the study. IM, DB, AB, HM, DD, SV, DR, EC, DG, KH, AI, NF, VQM, NT, JL, FU, SGL, AB, IKP and VH recruited and phenotyped the patients. MR and GS conducted the analyses and interpreted the results. MR, GB and GS drafted, the manuscript. MR, GB and IT edited and prepared the final version of the manuscript.

\section{Compliance with ethical standards}

Conflict of interest The authors declare that they have no conflict of interest.

\section{References}

1. Zhou Q, Yang D, Ombrello AK, et al. Early-onset stroke and vasculopathy associated with mutations in ADA2. N Engl J Med. 2014;370:911-20.

2. Navon Elkan P, Pierce SB, Segel R, et al. Mutant adenosine deaminase 2 in a polyarteritis nodosa vasculopathy. N Engl J Med. 2014;370:921-31.

3. Caorsi R, Penco F, Schena F, Gattorno M. Monogenic polyarteritis: the lesson of ADA2 deficiency. Pediatr Rheumatol Online J. 2016;14:14 https://doi.org/10.1186/s12969-016-0111-7

4. Oda H, Kastner DL. Genomics, biology, and human illness: advances in the monogenic autoinflammatory diseases. Rheum Dis Clin N Am. 2017;43:327-45.

5. Hashem H, Kelly SJ, Ganson NJ, Hershfield MS. Deficiency of adenosine deaminase 2 (DADA2), an inherited cause of polyarteritis nodosa and a mimic of other systemic rheumatologic disorders. Curr Rheumatol Rep. 2017;19:70.

6. Gonzalez Santiago TM, Zavialov A, Saarela J, et al. Dermatologic features of ADA2 deficiency in cutaneous polyarteritis nodosa. JAMA Dermatol. 2015;151:1230-4.

7. Schepp J, Bulashevska A, Mannhardt-Laakmann W, et al. Deficiency of adenosine deaminase 2 causes antibody deficiency. J Clin Immunol. 2016;36:179-86.

8. Schepp J, Proietti M, Frede N, et al. Screening of 181 patients with antibody deficiency for deficiency of adenosine deaminase 2 sheds new light on the disease in adulthood. Arthritis Rheumatol. 2017;69:1689-700.
9. Van Montfrans JM, Hartman EAR, Braun KPJ, et al. Phenotypic variability in patients with ADA2 deficiency due to identical homozygous R169Q mutations. Rheumatology. 2016;55: 902-10.

10. Van Eyck L, Hershfield MS, Pombal D, et al. Hematopoietic stem cell transplantation rescues the immunologic phenotype and prevents vasculopathy in patients with adenosine deaminase 2 deficiency. J Allergy Clin Immunol. 2015;135:283-7.e5.

11. Zavialov AV, Gracia E, Glaichenhaus N, Franco R, Zavialov AV, Lauvau G. Human adenosine deaminase 2 induces differentiation of monocytes into macrophages and stimulates proliferation of $\mathrm{T}$ helper cells and macrophages. J Leukoc Biol. 2010;88:279-90.

12. Zavialov AV, Yu X, Spillmann D, Lauvau G, Zavialov AV. Structural basis for the growth factor activity of human adenosine deaminase ADA2 $\bullet$. J Biol Chem. 2010;285:12367-77.

13. Zavialov AV, Engström A. Human ADA2 belongs to a new family of growth factors with adenosine deaminase activity. Biochem J. 2005;391:51-57.

14. Belot A, Wassmer E, Twilt M, et al. Mutations in CECR1 associated with a neutrophil signature in peripheral blood. Pediatr Rheumatol. 2014;12:44.

15. Poswar F de O, da Fonseca RMT, de Albuquerque LCP, et al. Adenosine deaminase 2 deficiency presenting as spastic paraplegia and systemic vasculitis. J Neurol. 2016;263:818-20.

16. Nanthapisal S, Murphy C, Omoyinmi E, et al. Deficiency of adenosine deaminase type 2: a description of phenotype and genotype in fifteen cases. Arthritis Rheumatol. 2016;68:2314-22.

17. Westendorp WF, Nederkoorn PJ, Aksentijevich I, Hak AE, Lichtenbelt KD, Braun KPJ. Unexplained early-onset lacunar stroke and inflammatory skin lesions: consider ADA2 deficiency. Neurology. 2015;84:2092-3.

18. Uettwiller F, Sarrabay G, Rodero MP, et al. ADA2 deficiency: case report of a new phenotype and novel mutation in two sisters. RMD Open. 2016;2:e000236.

19. Touitou I, Lesage S, McDermott M, et al. Infevers: an evolving mutation database for auto-inflammatory syndromes. Hum Mutat. 2004;24:194-8.

20. Caorsi R, Penco F, Grossi A, et al. ADA2 deficiency (DADA2) as an unrecognised cause of early onset polyarteritis nodosa and stroke: a multicentre national study. Ann Rheum Dis. 2017;76:1648-56. https://doi.org/10.1136/annrheumdis-2016210802

21. Van Eyck L, Liston A, Wouters C. Mutant ADA2 in vasculopathies. N Engl J Med. 2014;371:480.

22. Fellmann F, Angelini F, Wassenberg J, et al. IL-17 receptor A and adenosine deaminase 2 deficiency in siblings with recurrent infections and chronic inflammation. J Allergy Clin Immunol. 2016;137:1189.e2-96.e2.

23. Federici S, Sormani MP, Ozen S, et al. Evidence-based provisional clinical classification criteria for autoinflammatory periodic fevers. Ann Rheum Dis. 2015;74:799-805.

24. Batu ED, Karadag O, Taskiran EZ, et al. A case series of adenosine deaminase 2-deficient patients emphasizing treatment and genotype-phenotype correlations. J Rheumatol. 2015;42:1532-4.

\section{Affiliations}

Mélanie Rama ${ }^{1} \cdot$ Claire Duflos $^{2} \cdot$ Isabelle Melki,4 ${ }^{3,4}$ Didier Bessis $^{5} \cdot$ Axelle Bonhomme $^{6} \cdot$ Hélène Martin $^{6}$. Diane Doummar $^{7,8} \cdot$ Stéphanie Valence $^{7}$ - Diana Rodriguez ${ }^{7,8}$ - Emilie Carme ${ }^{9}$ - David Genevieve ${ }^{10,11}$.

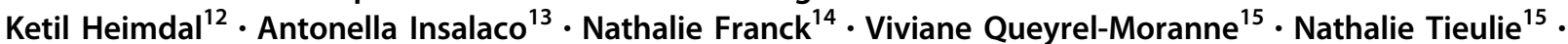
Jonathan London ${ }^{16}$. Florence Uettwiller ${ }^{17}$. Sophie Georgin-Lavialle ${ }^{18} \cdot$ Alexandre Belot $^{19} \cdot$ Isabelle Koné-Paut $^{20}$. Véronique Hentgen ${ }^{21} \cdot$ Guilaine Boursier $^{1} \cdot$ Isabelle Touitou $\mathbb{D}^{1,10} \cdot$ Guillaume Sarrabay $^{1,10}$ 
1 Laboratory of Rare and Autoinflammatory Genetic Diseases and CEREMAIA, Montpellier University Hospital, Montpellier, France

2 Medical Information Department, Montpellier University Hospital, Montpellier, France

3 General Pediatrics, Infectious Disease and Internal Medicine Department, AP-HP, Robert Debré Hospital, Paris, France

4 Pediatric Hematology-Immunology and Rheumatology Department, AP-HP, Necker-Enfants Malades Hospital, Paris, France

5 Department of Dermatology, Saint-Eloi Hospital and Montpellier University Hospital, Montpellier, France

6 Department of Dermatology, Hôpital d'Instruction des Armées Legouest, Metz, France

7 Pediatric Neurology and National Reference Center for Neurogenetic Disorders, AP-HP, Trousseau Hospital, Paris, France

8 GRC ConCer-LD, Sorbonne Universites, UPMC Universite, Paris, France

9 Neuropediatrics, Gui de Chaulliac Hospital, Montpellier University Hospital, Montpellier, France

10 Genetics and Immunopathology of Inflammatory Osteoarticular Diseases, INSERM UMR1183, Montpellier, France
11 Department of Clinical Genetics, Montpellier University Hospital, Montpellier, France

12 Department of Medical Genetics, Oslo University Hospital, Oslo, Norway

13 Division of Rheumatology, IRCCS Pediatrico Bambino Gesù Children's Hospital, Roma, Italy

14 Department of Dermatology, Teaching Hospital Cochin, AP-HP, University Paris Descartes, Paris, France

15 Internal Medicine, Archet-1 Hospital, Nice University Hospital, Nice, France

16 Internal Medecine, Cochin Hospital, AP-HP, Paris Descartes University, Paris, France

17 Pediatric Immunology-Hematology and Rheumatology Unit, Institut Imagine, AP-HP, Necker Enfants Malades Hospital, APHP, Paris, France

18 Internal Medicine, CEREMAIA, Tenon Hospital, AP-HP, University of Pierre et Marie Curie, Paris, France

19 Department of Paediatric Nephrology, Rheumatology and Dermatology, RAISE, Lyon University Hospital, Lyon, France

20 Department of Paediatric Rheumatology, CEREMAIA, Bicêtre Hospital, AP-HP, University of Paris Sud, Le Kremlin-Bicêtre, France

21 Department of Paediatrics, CEREMAIA, Versailles Hospital, Versailles, France 